\title{
Concordancia entre el diagnóstico clínico y el patológico por necropsias
}

\author{
Edith Valdez-Martínez, M.C., M. en C., (1) Ernesto Arroyo-Lunagómez, M.C., (2)
}

Luis Landero-López, M.C.(3)

\begin{abstract}
Valdez-Martínez E, Arroyo-Lunagómez E, Landero-López L. Concordancia entre el diagnóstico clínico y el patológico por necropsias. Salud Pública Mex 1998;40:32-37.
\end{abstract}

\section{Resumen}

Objetivo. Determinar la concordancia entre diagnósticos clínicos y patológicos con base en los resultados de necropsias. Material y métodos. Se revisaron 67 protocolos de necropsia y expedientes clínicos de niños y adultos del servicio de patología del Centro de Especialidades Médicas del Estado de Veracruz Doctor Rafael Lucio, durante 1995 y 1996. Se obtuvieron las frecuencias simples de las variables de estudio, y la concordancia se calculó con el estadístico kappa simple. Resultados. En general la cifra de necropsias fue de $10.5 \%$. Correspondió a la población infantil la mayor frecuencia (I7.5\%). El $59 \%$ de diagnósticos clínicos en niños y el $57 \%$ en adultos presentaron algún tipo de error. La concordancia del diagnóstico principal ante y postmortem fue de $26 \%$ (infantes) y $41 \%$ (adultos). Conclusiones. La alta cifra de errores diagnósticos y la consecuente baja concordancia clínico-patológica apoyan el hecho de que, a pesar del avance tecnológico en los procedimientos diagnósticos, es necesario realizar necropsias con el fin de documentar de manera más definitiva el control de calidad del ejercicio de la medicina.

Palabras clave: errores diagnósticos; diagnóstico clínico; patología; México

\author{
Valdez-Martínez E, \\ Arroyo-Lunagómez E, Landero-López L. \\ Clinico-pathological \\ concordance at autopsies. \\ Salud Publica Mex 1998;40:32-37.
}

\section{Abstract}

Objective. To determine the degree of concordance between clinical and autopsy diagnosis. Material and methods. The records of 67 autopsies and clinical files of children and adults, comprising the years 1995 to 1996, from a third level hospital in the State of Veracruz, Mexico, were revised. The concordance score was calculated with the statistical kappa. Results. In general, the rate of autopsy was $10.5 \%$, with higher frequency for children (17.5\%). In children, $59 \%$ of clinical diagnoses revealed mistakes and in adults, $57 \%$. The degree of agreement in the underlying cause ante and postmortem was $26 \%$ in children and $41 \%$ in adults. Conclusions. The high rate of diagnostic error and low concordance score between clinical and autopsy diagnosis strongly suggests that, in spite of increased availability of modern diagnostic techniques, the necessity of postmortem studies to control the level of medical practice is evident.

Key words: diagnostic error; diagnosis, clinical; pathology; Mexico

(I) Departamento de Investigación Médica, Centro de Especialidades Médicas del Estado de Veracruz Dr. Rafael Lucio (CEMEV), México.

(2) Departamento de Medicina Interna, CEMEV, México.

(3) Departamento de Anatomía Patológica, CEMEV, México. 
E s universalmente aceptado el gran valor de los estudios postmortem; ${ }^{1}$ empero, en los últimos 40 años ha ocurrido una notable disminución de los mismos, tanto en el ámbito mundial ${ }^{2-5}$ como en la República mexicana. ${ }^{1,4}$ Son varios los argumentos que hacen que el estudio postmortem sea considerado erróneamente como un procedimiento anticuado y obsoleto, ${ }^{6}$ señalándose como responsables a los obstáculos legales, prejuicios sociales y religiosos de los familiares, resistencia de los administradores por el alto costo del método, inclinación de los anatomopatólogos hacia la patología quirúrgica o experimental, temor de algunos médicos de exponer su prestigio ante los hallazgos de necropsia y al exceso de confianza en la nueva tecnología para diagnóstico. ${ }^{1-4,7,8}$ Bajo este contexto resulta importante mencionar que los avances tecnológicos, por ejemplo, las imágenes por resonancia magnética y las pruebas genéticas, han aumentado nuestra certeza en el diagnóstico; además, la incorporación de pruebas diagnósticas "con números rigurosos" han llevado a una confianza comprensible en el laboratorio clínico; sin embargo, los diagnósticos suelen ser escasos, parciales, erróneos o contradictorios. ${ }^{9,} 10$ Esta afirmación coincide con lo referido por algunos investigadores, quienes consideran que la certidumbre absoluta en el diagnóstico clínico es casi inalcanzable.9,11

Se sabe que aun en los hospitales de gran prestigio ocurre un porcentaje grande de errores, a juzgar por la falta de coincidencia entre el diagnóstico clínico y el diagnóstico definitivo proporcionado por la necropsia; las cifras varían de 20 a 60\%, incluso en los casos de enfermedades comunes. ${ }^{1,2,12}$ Por ende, la correlación clínico-patológica que se logra a través de las necropsias resulta ser de gran valor no sólo para determinar la causa de muerte y proyectar estadísticas de mortalidad confiables, ${ }^{2,12,13}$ sino que, además, permite evaluar la calidad de la atención médica ${ }^{14} \mathrm{y}$, sobre todo, su trascendencia en la educación médica continua de pre y posgrado., 2,14,15 Por lo tanto, el objetivo del presente trabajo fue cuantificar la concordancia existente entre los diagnósticos clínicos y los patológicos con base en los resultados de necropsias realizadas en un hospital de tercer nivel.

\section{Material y métodos}

Se realizó un estudio transversal, descriptivo y retrolectivo (encuesta hospitalaria). Fueron evaluados un total de 67 expedientes clínicos y protocolos de necropsia de niños y adultos, correspondientes a los años de 1995 y 1996, del servicio de patología del Centro de Especialidades Médicas Doctor Rafael Lucio (CEMEV), organismo descentralizado del gobierno del estado de
Veracruz, el cual funciona como un centro de tercer nivel de atención médica, con 148 camas censables.

Las variables estudiadas fueron edad, sexo, tiempo de hospitalización, diagnósticos clínicos y patológicos y errores diagnósticos; estos últimos identificados y tipificados de acuerdo con la clasificación de Goldman, Cameron y McGoogan;3,16 la información se registró en una hoja de recolección de datos, con previa prueba piloto y estandarización de criterios operativos.

Los diagnósticos clínicos que carecían de expresión morfológica fueron considerados como correctos; por ejemplo, diabetes mellitus no complicada de reciente instalación, cetoacidosis, etcétera. ${ }^{12}$ Se consideraron hallazgos de necropsia las entidades o condiciones que no fueron diagnosticadas en vida. ${ }^{12}$

Conviene hacer notar que a la enfermedad, lesión o complicación que causó directamente el fallecimiento, se le identificó como causa directa de muerte. ${ }^{3}$ El diagnóstico principal o enfermedad primaria correspondió a todas las entidades nosológicas que condicionaron la causa directa de muerte. ${ }^{3}$ Fueron englobados como diagnósticos menores relacionados con la enfermedad primaria todos aquellos relacionados con el diagnóstico principal, pero no directamente con la causa de muerte. ${ }^{3}$ También fueron identificados los diagnósticos menores no relacionados con la enfermedad primaria, es decir, aquellos padecimientos no relacionados con la enfermedad principal pero que eventualmente pudieron haber contribuido a la muerte; ${ }^{3}$ así, por ejemplo, se puede identificar un paciente que murió por septicemia (causa directa) secundaria a pielonefritis (diagnóstico principal), además de ser portador de diabetes mellitus (diagnóstico menor relacionado) y de cardiopatía isquémica (diagnóstico menor no relacionado); de esta forma se cuantificó el grado de acuerdo anatomoclínico, a través del estadístico kappa simple. ${ }^{17}$

Para el análisis del error diagnóstico se formaron las siguientes categorías: ${ }^{3,16}$ clase I, cuando la detección oportuna hubiera condicionado cambio en el manejo y probable cura o sobrevida prolongada (p.e., tuberculosis pulmonar); clase II, cuando la detección oportuna no hubiera condicionado cambio en el manejo (p.e., cirrosis hepática); clase III, en los casos de no detección de enfermedades relacionadas con la enfermedad principal, pero no directamente con la causa de muerte (p.e., trombosis intramural en pacientes con insuficiencia cardiaca), y clase IV, los diagnósticos no relacionados con la enfermedad principal y que ocasionalmente pudieron haber afectado el pronóstico; también quedan incluidos los procesos que contribuyeron a la muerte en pacientes con enfermedad terminal (p.e., broncoaspiración en pacientes con neumonía y leucemia). 
En el análisis se obtuvieron las frecuencias simples de las diferentes variables; el grado de concordancia se calculó con kappa simple ${ }^{17}$ para lo cual se clasificaron por separado niños y adultos. Una vez obtenidos los valores kappa, éstos se interpretaron siguiendo los lineamientos de Landis y Koch. ${ }^{18}$ Para la captura y el análisis de los datos se utilizó el paquete Epi Info versión 6.

\section{Resultados}

Durante el periodo de estudio se realizaron 75 necropsias completas de un total de 716 muertes, lo cual arroja una cifra de $10.5 \%$; fue posible obtener información en 67 de los estudios postmortem (89\%). La mayor cantidad de necropsias se realizó en el grupo infantil $(17.5 \%)$ (cuadro I).

\section{Población infantil}

La mediana de edad fue de 16.5 días con una amplitud de un día a 13 años; 52\% de los infantes fueron del sexo masculino.

De las 46 necropsias (completas) realizadas, 27 (58.7 \%) mostraron algún tipo de error en el diagnóstico clínico, pudiéndose constatar en el grupo de enfermedades infecciosas el mayor número de errores (cuadro II). Entre los hallazgos de necropsia predominaron los pertenecientes al grupo de enfermedades genitourinarias $(n=11)$, digestivas $(n=10)$ y respiratorias $(\mathrm{n}=7)$.

El 50\% de los estudios postmortem se realizaron en niños con cinco o más días de estancia hopsitalaria; cabe mencionar que los errores mayores fueron en sujetos que tuvieron una estancia más prolongada; esto es, la mediana de estancia intrahospitalaria para los errores clase I y II fue de nueve días, mientras que para los errores III y IV fue de un día.

El cuadro III incluye todos los diagnósticos. Al cuantificar la concordancia en forma global encon-

\section{Cuadro I}

NeCROPSIAS EN NIÑOS Y ADULTOS POR AÑO DE ESTUdIO. Centro de Especialidades Médicas del Estado de Veracruz Dr. Rafael Lucio. México, I 995-I 996

\begin{tabular}{lrrrrrr} 
Grupos de & \multicolumn{2}{c}{1995} & \multicolumn{2}{c}{1996} & \multicolumn{2}{c}{ Total } \\
población & NN/ND & $\%$ & NN/ND & $\%$ & NN/ND & $\%$ \\
& & & & & & \\
Niños & $14 / 86$ & 16.3 & $32 / 177$ & 18.1 & $46 / 263$ & 17.5 \\
\hline Adultos & $14 / 203$ & 6.9 & $7 / 205$ & 3.4 & $21 / 408$ & 5.1
\end{tabular}

$\mathrm{NN}=$ número de necropsias $\quad \mathrm{ND}=$ número de defunciones tramos un acuerdo observado de 31\%, el atribuible al azar de $26 \%$ y el valor de kappa de 0.07 , lo que se traduce en un acuerdo mínimo; las diferencias entre la concordancia observada y la atribuible al azar fueron estadísticamente significativas $(z=1.7, p=0.04)$. Al evaluar sólo los diagnósticos principales el tipo de acuerdo mejoró, encontrándose un valor kappa de 0.26 esti-

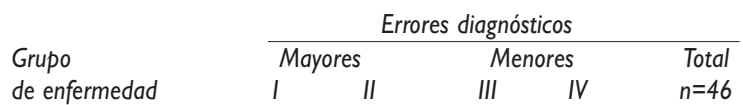

\begin{tabular}{lllllc} 
Infecciosas & 4 & 3 & 4 & 3 & 14 \\
\hline Neurológicas & 0 & 3 & $\mathrm{I}$ & 0 & 4 \\
\hline Cardiovasculares & 0 & 2 & 0 & 0 & 2 \\
\hline Digestivas & 0 & 2 & 0 & 0 & 2 \\
\hline Endocrinológicas & 0 & 0 & $\mathrm{I}$ & $\mathrm{I}$ & 2 \\
\hline Neoplásicas & $\mathrm{I}$ & $\mathrm{I}$ & 0 & 0 & 2 \\
\hline Respiratorias & 0 & 0 & $\mathrm{I}$ & 0 & $\mathrm{I}$ \\
Total & & & & & \\
\hline
\end{tabular}

Cuadro III

CONCORDANCIA CLÍNICO-PATOLÓGICA POR NECROPSIAS en niños. Centro de Especialidades Médicas del Estado de Veracruz Dr. Rafael Lucio. MÉXıCO, I995-I996

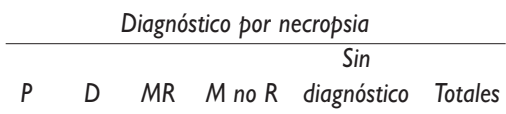

\begin{tabular}{|c|c|c|c|c|c|c|c|}
\hline & $P$ & 36 & 0 & 0 & 0 & 18 & 54 \\
\hline Diagnóstico & $D$ & 0 & 21 & I & 0 & 33 & 55 \\
\hline \multirow[t]{3}{*}{ clínico } & $M R$ & 0 & 0 & 7 & 0 & 6 & 13 \\
\hline & $M$ no $R$ & 0 & 0 & I & 0 & 3 & 4 \\
\hline & agnóstico & 24 & 26 & 21 & 12 & 0 & 83 \\
\hline & Totales & 60 & 47 & 30 & 12 & 60 & 209 \\
\hline
\end{tabular}

$\mathrm{P}=$ diagnósticos principales

$\mathrm{D}=$ causas directas de muerte

$M R=$ diagnósticos menores relacionados

$M$ no $R=$ diagnósticos menores no relacionados 
mado como acuerdo regular. Sin embargo, cuando se analizaron únicamente las causas directas de muerte el valor kappa fue de 0.17 considerado como acuerdo mínimo.

\section{Población adulta}

La mediana de edad fue de 37 años con una amplitud de 19 a 65 años; $62 \%$ de los sujetos fueron del sexo masculino.

De las 21 necropsias evaluadas, 12 (57\%) clasificaron al diagnóstico clínico con algún tipo de error (cuadro IV). La mayor frecuencia de errores diagnósticos se identificó en los grupos de enfermedades respiratorias e infecciosas.

Dentro de los hallazgos de necropsia destacan los del grupo de enfermedades digestivas $(n=12)$, genitourinarias $(n=10)$ y respiratorias $(n=6)$.

En relación con el tiempo de hospitalización, éste tuvo una mediana de siete días con una amplitud que varió de uno a 30 días, sin que predominara una moda para un tipo de error específico.

La tabulación global de los diagnósticos se muestra en el cuadro V; el análisis de los mismos dio una concordancia observada de 39\%; la atribuible al azar se calculó en $17 \%$, con un valor de kappa de 0.23 , lo que indica un acuerdo regular; las diferencias entre concordancia observada y la atribuible al azar fueron estadísticamente significativas $\left(z=5.21, p=0 \times 10^{-6}\right)$. Al evaluar únicamente los diagnósticos principales y las causas directas de muerte, por separado, la concordancia se elevó (valor kappa) a 0.41 y 0.31 , respectivamente, lo cual traduce un acuerdo regular.

\section{Discusión}

La literatura mundial revela un porcentaje de necropsias que oscila entre 22 y $38 \%, 2,3$ sin embargo, en algunos hospitales del norte de Irlanda, por ejemplo, las cifras varían de 2 a $6 \%{ }^{2}$ Actualmente, en la República mexicana $26.1 \%$ de las necropsias se lleva a cabo en el Distrito Federal, y 13.5\% en el resto del país. ${ }^{19}$

Existen varias opiniones acerca de la cantidad ideal de necropsias para un hospital general; empero, la mayoría coincide en que una cifra superior a $20 \%$ representa un número adecuado de casos para la enseñanza de pre y posgrado, para la educación médica continua y para la evaluación de la atención médica. ${ }^{20}$

Lo anterior pone de manifiesto la baja tasa de necropsias $(10.5 \%)$ realizadas en nuestro hospital.

Es preponderante mencionar que el interés central de este informe fue identificar un parámetro cuantitativo que nos permita evaluar la calidad de la atención médica, a través de la medición de la competencia clínica como un todo, por lo que el número de necropsias incluidas resulta útil.

Cuando la población de estudio fue dividida en adulta y pediátrica encontramos un predominio de estudios anatomo-patológicos en la población infantil, sin que esto fuese debido a la inclusión de óbitos; esta situación prevalece en la mayoría de los hospitales, ${ }^{2}$ donde la población pediátrica pareciera ser un área en

\begin{tabular}{|c|c|c|c|c|}
\hline \multicolumn{5}{|c|}{ 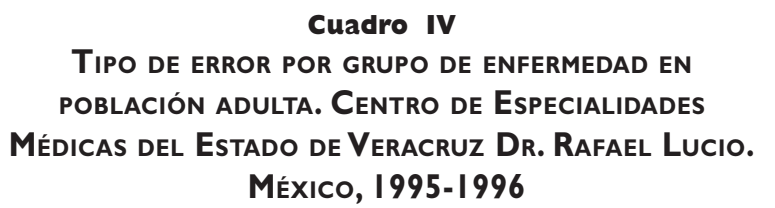 } \\
\hline \multirow[b]{2}{*}{$\begin{array}{l}\text { Grupo } \\
\text { de enfermedad }\end{array}$} & \multicolumn{4}{|c|}{ Errores diagnósticos } \\
\hline & $\begin{array}{c}\text { Mayores } \\
\text { II }\end{array}$ & ${ }_{I I I}^{\Lambda}$ & s & $\begin{array}{l}\text { Total } \\
n=21\end{array}$ \\
\hline Respiratorias & 2 & 2 & 0 & 4 \\
\hline Infecciosas & 2 & I & 0 & 3 \\
\hline Digestivas & 0 & I & 2 & 3 \\
\hline Genitourinarias & I & 0 & 0 & I \\
\hline Neurológicas & 0 & I & 0 & I \\
\hline Total & 5 & 5 & 2 & 12 (57.0\%) \\
\hline
\end{tabular}

\section{Cuadro V}

CONCORDANCIA CLÍNICO-PATOLÓGICA POR NECROPSIAS en población adulta. Centro de Especialidades Médicas del Estado de Veracruz Dr. Rafael Lucio. MÉXıCO, I 995 - 1996

\begin{tabular}{|c|c|c|c|c|c|c|}
\hline & \multicolumn{6}{|c|}{ Diagnóstico por necropsia } \\
\hline & \multirow[b]{2}{*}{$P$} & \multirow[b]{2}{*}{ D } & \multirow[b]{2}{*}{$M R$} & \multicolumn{3}{|c|}{ Sin } \\
\hline & & & & $M$ no $R$ & diagnóstico & Totales \\
\hline$P$ & 19 & 0 & 0 & 0 & 6 & 25 \\
\hline Diagnóstico & 0 & 17 & $\mathrm{I}$ & 0 & 10 & 28 \\
\hline clínico & 0 & 0 & 4 & 0 & I & 5 \\
\hline$M$ no $R$ & 0 & 0 & 0 & 4 & 3 & 7 \\
\hline Sin diagnóstico & 10 & 7 & 14 & 17 & 0 & 48 \\
\hline Totales & 29 & 24 & 19 & 21 & 20 & 113 \\
\hline \multicolumn{7}{|c|}{$\mathrm{P}=$ diagnósticos principales } \\
\hline \multicolumn{7}{|c|}{$D=$ causas directas de muerte } \\
\hline \multicolumn{7}{|c|}{$M R=$ diagnósticos menores relacionados } \\
\hline \multicolumn{7}{|c|}{ M no $R=$ diagnósticos menores no relacionados } \\
\hline
\end{tabular}


la cual las necropsias permanecen como un procedimiento trascendente.

En cuanto a la distribución por sexo, nuestros resultados tienen consistencia con lo publicado, ${ }^{1,19,}, 20$ en ambas poblaciones la cifra de necropsias en mujeres fue menor que en hombres. Respecto a los grupos de edad, en los niños las mayores frecuencias correspondieron a los neonatos, mientras que en los adultos las cifras menores fueron para los sujetos de edad avanzada. Esto último coincide con lo observado en otros hospitales; ${ }^{1}$ esto es, cuando el adulto mayor muere, por lo general no se concede la autorización y, al mismo tiempo, la necropsia se solicita menos frecuentemente.

En lo referente a los errores diagnósticos, las cifras oscilan entre 6 y $68 \% .{ }^{3,21}$ Lo anterior coloca nuestros hallazgos (59 y $57 \%$ en niños y en adultos, respectivamente) dentro de los más altos; sin embargo, debemos considerar la posibilidad de una sobrestimación por el hecho de que en el CEMEV, como en otros hospitales, ${ }^{21}$ la mayoría de las solicitudes de necropsia se limitan a pacientes que cursaron con elevada incertidumbre diagnóstica; por otra parte, conviene mencionar que en las enfermedades infecciosas (niños) y en las respiratorias (adultos) se presentó la mayor frecuencia de errores diagnósticos. Esto último tiene consistencia con lo referido por otros investigadores; ${ }^{3,13}$ además, es más fácil obtener el consentimiento de los familiares cuando se trata de un padecimiento infeccioso que cuando se trata de una enfermedad degenerativa. $3,12,21$

Fue interesante encontrar una cantidad superior de errores mayores en la población pediátrica (59\%), mientras que en los adultos predominaron los errores menores (57\%). La existencia de errores diagnósticos trascendentes en más de la mitad de los casos de necropsia no implica que en el CEMEV sean más frecuentes los errores que los aciertos. De acuerdo con otros autores, ${ }^{13,22}$ estas cifras alarmantes tienen una serie de atenuantes si consideramos, por un lado, que los casos en los que se hace la necropsia son aquellos particularmente difíciles, tanto por la complejidad de la enfermedad en sí como por el grado de avance de la misma; por otro lado, debe considerarse que en un buen número de casos, el diagnóstico correcto y el tratamiento adecuado curan la enfermedad, y los pacientes son dados de alta sanos o al menos controlados. En el mismo sentido apunta la explicación al mínimo acuerdo clínicopatológico, kappa $=7 \%$ (niños) y 23\% (adultos), al tomar en cuenta todas las causas de muerte. Obviamente, la concordancia anatomo-clínica se elevó a un acuerdo regular y bueno ${ }^{18}$ cuando se consideraron únicamente y por separado los diagnósticos principales (kappa $=26 \%$ en niños y $41 \%$ en adultos) y las causas directas de muerte (kappa $=17 \%$ en niños y $31 \%$ en adultos). La alta cantidad de errores diagnósticos y, por consecuencia, el mínimo acuerdo clínico-patológico, aunados al antecedente de por lo menos siete días de estancia intrahospitalaria, ponen de manifiesto una vez más ${ }^{21}$ que la interconsulta con los especialistas y la moderna tecnología diagnóstica no mejoran en mucho la certeza del diagnóstico, ${ }^{13}$ y demuestra que los estudios postmortem son el mejor medio para establecer, confirmar, aclarar y corregir errores diagnósticos. ${ }^{1,4,6,12,16}$ Por lo tanto, el ejercicio de una buena medicina no puede soslayar este procedimiento, ya que a través del mismo, de manera directa e indirecta, se afinan los diagnósticos, y esto redunda en beneficio del paciente y en el aprendizaje para el médico.

En realidad, en esta era de la biología molecular el estudio postmortem no ha dejado de ser útil; por el contrario, la utilización de la nueva metodología en los estudios anatomo-patológicos contribuye a incrementar el conocimiento médico, de tal manera que con la realización sistemática de la necropsia podemos mejorar nuestras habilidades clínicas y determinar si estamos progresando en la reducción de nuestra tasa de discrepancia clínica.

\section{Referencias}

I.Aguirre GJ. Simposio. La autopsia: ¿un procedimiento que ha pasado de moda? Gac Med Mex 1988;124:339-357.

2. Anderson NH, Shanks JH, McCluggage GWG, Toner PG. Necropsies in clinical audit. J Clin Pathol 1989;42:897-90I.

3. Goldman L, Sayson R, Robbins S, Cohn LH, Bettmann M,Weisberg M. The value of the autopsy in three medical areas. N Engl J Med I983;308: I0001005.

4. Contreras RR. Simposio. La autopsia. Gac Med Mex 1976; I I I: I-4. 5. McPhee SJ. Maximizing the benefits of autopsy for clinicians and families. What needs to be done. Arch Pathol Lab Med 1996;120:1 I64-II65.

6. Benítez BL. La autopsia y la medicina moderna. Gac Med Mex 1988; 124:352357.

7. Start RD. Risk management, loss control and the autopsy. Br J Hosp Med 1993;50:576-578.

8. McManus BM, Wood SM.The autopsy. Simple thoughts about the public needs and how to address them. Am J Clin Pathol 1996; 106: I IS-14S.

9. Sackett DL, Haynes RB, Guyatt GH, Tugwell P. Epidemiología clínica. Ciencia básica para la medicina clínica. 2a. edición. Buenos Aires, Argentina: Editorial Panamericana, 1994:35-6I, 87-II8.

10. Kassirer JP. Sounding board, our stubborn quest for diagnostic certainty. N Engl J Med 1989;320: I489-I49I.

II. Kassirer JP. Diagnostic reasoning. Ann Intern Med 1989;1 10:893-900. 12. Angeles AA, Quintanilla ML, Muñoz FL, Espinoza VB, Victoria PP. Concordancia diagnóstica clínico-patológica en 429 autopsias del Instituto Nacional de la Nutrición Salvador Zubirán. Rev Invest Clin 1992;44:13-20.

13. Szende B, Kendrey G, Lapis K, Lee PN, Roe FJC.Accuracy of admission and preautopsy clinical diagnoses in the light of autopsy findings: A study conducted in Budapest. Hum Exp Toxicol 1994; I3:67|-680. 
14. Start RD, Underwood JCE. Funding the clinical autopsy. J Pathol 1995; 177:5-9.

15. Velázquez T, Márquez MH. Manual de autopsias y piezas quirúrgicas. México, D.F.: Editorial La Prensa Médica Mexicana, 1968:1-10.

16. Cameron HM, McGoogan E. A prospective study of I I52 hospital autopsies. I. Innacuracies in death certification.J Pathol I 98I; I33:273-283. 17. Fajardo GA, Yamamoto KLT, Garduño EJ, Hernández HD, Martínez GC. Consistencia y validez de una medición en la investigación clínica pediátrica. Definición, evaluación y su interpretación. Bol Med Hosp Infant Mex |99|;48:367-38|.
18. Landis RJ, Koch GG. The measurement of observer agreement for categorical data. Biometrics 1977;33:159-174.

19. Alonso P. Autopsias en los hospitales de enseñanza en México. Gac Med Mex 1988; 124:349-352.

20.Aguirre GJ.Panorama actual de las autopsias. Gac Med Mex 1988; 124:347349.

21. Britton M. Diagnostic errors discovered at autopsy. Acta Med Scand 1974;196:203-210. 